\title{
Chromosome 7 gain and DNA hypermethylation at the HOXA10 locus are associated with expression of a stem cell related HOX-signature in glioblastoma
}

\author{
Sebastian Kurscheid ${ }^{1,2,3,16}$, Pierre Bady ${ }^{1,2,3,4}$, Davide Sciuscio ${ }^{1,2}$, Ivana Samarzija ${ }^{1,2}$, Tal Shay $^{5}$, Irene Vassallo, ${ }^{1,2}$, \\ Wim V Criekinge ${ }^{6}$, Roy T Daniel ${ }^{1}$, Martin J van den Bent ${ }^{7}$, Christine Marosi $^{8}$, Michael Weller ${ }^{9,10}$, Warren P Mason ${ }^{11}$, \\ Eytan Domany ${ }^{12}$, Roger Stupp ${ }^{1,13}$, Mauro Delorenzi ${ }^{3,14,15}$ and Monika E Hegi ${ }^{1,2^{*}}$
}

\begin{abstract}
Background: HOX genes are a family of developmental genes that are expressed neither in the developing forebrain nor in the normal brain. Aberrant expression of a HOX-gene dominated stem-cell signature in glioblastoma has been linked with increased resistance to chemo-radiotherapy and sustained proliferation of glioma initiating cells. Here we describe the epigenetic and genetic alterations and their interactions associated with the expression of this signature in glioblastoma.
\end{abstract}

Results: We observe prominent hypermethylation of the HOXA locus 7p15.2 in glioblastoma in contrast to non-tumoral brain. Hypermethylation is associated with a gain of chromosome 7, a hallmark of glioblastoma, and may compensate for tumor-driven enhanced gene dosage as a rescue mechanism by preventing undue gene expression. We identify the CPG island of the HOXA10 alternative promoter that appears to escape hypermethylation in the HOX-high glioblastoma. An additive effect of gene copy gain at 7p15.2 and DNA methylation at key regulatory CpGs in HOXA10 is significantly associated with HOX-signature expression. Additionally, we show concordance between methylation status and presence of active or inactive chromatin marks in glioblastoma-derived spheres that are HOX-high or HOX-low, respectively.

Conclusions: Based on these findings, we propose co-evolution and interaction between gene copy gain, associated with a gain of chromosome 7, and additional epigenetic alterations as key mechanisms triggering a coordinated, but inappropriate, HOX transcriptional program in glioblastoma.

\section{Background}

Glioblastoma (GBM) is an aggressive brain tumor with a median survival of only 15 months. Despite remarkable efforts targeting prominent pathogenetic biological features of GBM, efficacy of novel drugs has been disappointing and significant gains in overall survival have not been made since the introduction of combined radio-chemotherapy comprising TMZ [1]. GBM are notorious for their treatment resistance. This has been

\footnotetext{
* Correspondence: monika.hegi@chuv.ch

'Neurosurgery, Lausanne University Hospital, Lausanne 1011, Switzerland ${ }^{2}$ Neuroscience Research Center, Lausanne University Hospital, Lausanne 1011, Switzerland

Full list of author information is available at the end of the article
}

attributed to the deregulation of major tumor suppressing and oncogenic pathways [2], tumor heterogeneity [3], and exhibition of stem cell-like properties by so called tumor stem cells, or glioma initiating cells (GICs) [4]. GICs represent a subpopulation(s) of tumor cells and are believed to, first, give rise to tumor progeny due to their self-renewing capacities, and second, resist radio- and chemotherapy $[5,6]$.

In line with the notion of GICs' contribution to treatment resistance, we earlier reported a self-renewal-related, $H O X$-dominated gene expression signature in GBM associated with significantly worse outcome in patients homogenously treated in a clinical trial with combined 
chemo-radiotherapy comprising the alkylating agent temozolomide. This association was independent of the predictive effect of $M G M T$ methylation or age [7]. The abnormal expression of a $H O X$ gene signature has been confirmed recently in GICs, where it has been functionally associated with their glioma initiating potential [8]. The importance of $H O X$ gene expression for gliomagenesis and treatment resistance to temozolomide has been emphasized in several studies [8-12]. In 2006, Krivtsov and colleagues first described the inappropriate expression of a $H O X$ gene signature in acute myeloid leukemia (AML) [13]. The authors showed in an elegant experimental mouse model that acquisition of this stem cell related $H O X$ gene signature was associated with MLL-A9 fusion gene-induced leukemogenesis from committed progenitors of the granulocyte lineage, demonstrating for the first time that acquisition of stem cell properties in committed progenitor cells can lead to tumorigenesis.

HOX genes are a highly conserved family of genes encoding homeodomain transcription factors that provide anterior and posterior axial coordinates to vertebrate embryos during development [14]. In mammals, there are four paralogous $H O X$ gene clusters organized on different chromosomes (CHRs). These gene clusters represent loci with extremely high gene density. In humans they are located on CHR7 (HOXA), CHR17 (HOXB), CHR12 (HOXC), and CHR2 (HOXD). The spatial organization of $H O X$ genes is reflected in a $5^{\prime}$-posterior to 3 -anterior expression along the embryonal axes, termed spatial colinearity. Hence, expression of HOXA913 is predominantly found in sites of the extremities, while HOXA1-2 expression has been confirmed in, for example, the hindbrain. Although $H O X$ genes are involved in the development of the hindbrain, other non$H O X$ homeobox genes regulate the development of the mid- and forebrain [15]. The forebrain comprises the ventricular and the subventricular zone, which harbors neural stem cells even in the adult brain, and has been proposed as origin of gliomas in the adult. Although this remains debated, mouse models have provided functional support [16].

Given that $H O X$ genes are neither implicated in the developmental program of the brain nor expressed in the region of the adult brain that is thought to give rise to gliomas, we speculate that the HOX-signature is acquired during gliomagenesis, contributing stem cell properties. However, the mechanisms underlying the observed aberrant activation of $H O X$ genes in GBM remain elusive. It has been proposed that the PI3K-pathway may be an important upstream regulator of HOXA9 expression that is part of the HOX-signature [10]. A more recent report considered the involvement of MLL $(K M T 2 A)$ in at least a subset of HOX-high expressing
GBM. However, given the limited correlation reported, additional driver mechanisms triggering inappropriate HOX gene expression need to be considered [8]. Previous works have described a remarkable correlation of gene expression levels with gene dosage modulated by pathogenic copy number changes in cancer [17]. Most prominent among the $H O X$ expression signature genes are $H O X A$ genes, as corroborated by other labs $[7,8,10]$. The HOXA locus is located on CHR7 (7p15.2) that is affected by a copy number gain in up to $80 \%$ of GBM [18]. Most interestingly, gain of CHR7 has been proposed recently as the evolutionary first driver event in the development of primary GBM together with loss of one copy of CHR10 [19]. CHR7 harbors a number of potential driver genes, among many passengers that through CHR7 gain associated overexpression may drive/contribute to gliomagenesis. Of these, Ozawa et al. proposed PDGF as a driver gene for primary GBM, based on computational and experimental considerations. Previously we reported a low, but significant correlation between gene copy number of the HOXA cluster and expression of the HOX-signature [7]. However, only $42 \%$ of this patient cohort was found to be HOX-high. We therefore hypothesized that additional regulatory mechanisms are required to explain the abnormal expression of HOXA genes, with downstream effects on other HOX-signature genes. Here we present a model explaining the aberrant expression of the HOXsignature in GBM integrating multidimensional molecular data, comprising gene expression, gene copy number, and DNA methylation.

\section{Results}

Correlation between DNA methylation and gene expression in clinical GBM samples

During gliomagenesis extensive epigenetic remodeling takes place, including global DNA hypomethylation and focused hypermethylation of promoter CpG-islands (CGIs) frequently silencing tumor suppressor genes. Recurrent gain of additional copies of CHR7 in GBM, often referred to as trisomy 7 in the literature, likely affects the HOXA locus that dominates the HOXsignature. The 'HOX-signature' refers to the signature identified previously [7], and comprises 21 genes covered by 22 probes of the HG-133Plus2.0 GeneChip (Additional file 1: Table S1). We hypothesized that epigenetic silencing mediated by methylation of CpGs in CGI-promoters of HOX-signature genes may compensate for tumor driven enhanced gene dosage as a rescue mechanism that could at least partially explain the differences in expression. In order to test this hypothesis we analyzed DNA methylation profiles of $59 \mathrm{GBM}$ of the $\mathrm{NCH}$ EOORTC cohort obtained on the Illumina Infinium HumanMethylation450 BeadChip platform (450k). Indeed 
we found significant differences in the mean methylation across the HOXA locus on CHR7 (27,130,000 to 27,250,000; hg19 UCSC), when comparing GBM to non-tumoral brain $(\mathrm{n}=4)$, with a generalized hypermethylation in the GBM samples as measured at $504450 \mathrm{k}$ probes spanning the locus $(P<0.001$, two-sided t-test; Additional file 1: Figures S1 and S2). DNA methylation of the HOXA locus was significantly associated with gain of CHR7 ( $P<0.001$, two-sided t-test), which may indicate compensation for increased gene dosage. In order to identify regulatory methylation patterns relevant for the expression of the whole HOX-signature the 400 probes annotated in CGIs of the 21 HOXsignature genes were subjected to principle component analysis. The top $100 \mathrm{CpG}$ probes were selected based on their ranked cumulative contribution to the inertia of the DNA methylation table, hence representing the dominant contributors to the overall variability of the observed DNA methylation. The top 100 probes were predominantly located in the promoter CGIs of HOXA genes on CHR7, followed by HOXD genes on CHR2, HOXC genes on CHR12, and PROMININ1 on CHR4 (Figure 1A and Additional file 2: Table S2, which contains mean correlation, $P$ and $\mathrm{q}$-values, and functional annotation for all 100 selected Infinium 450k probes for NCH_EORTC and TCGA data). The correlation between methylation of these $\mathrm{CpGs}$ and expression of each member of the HOX-signature was calculated.

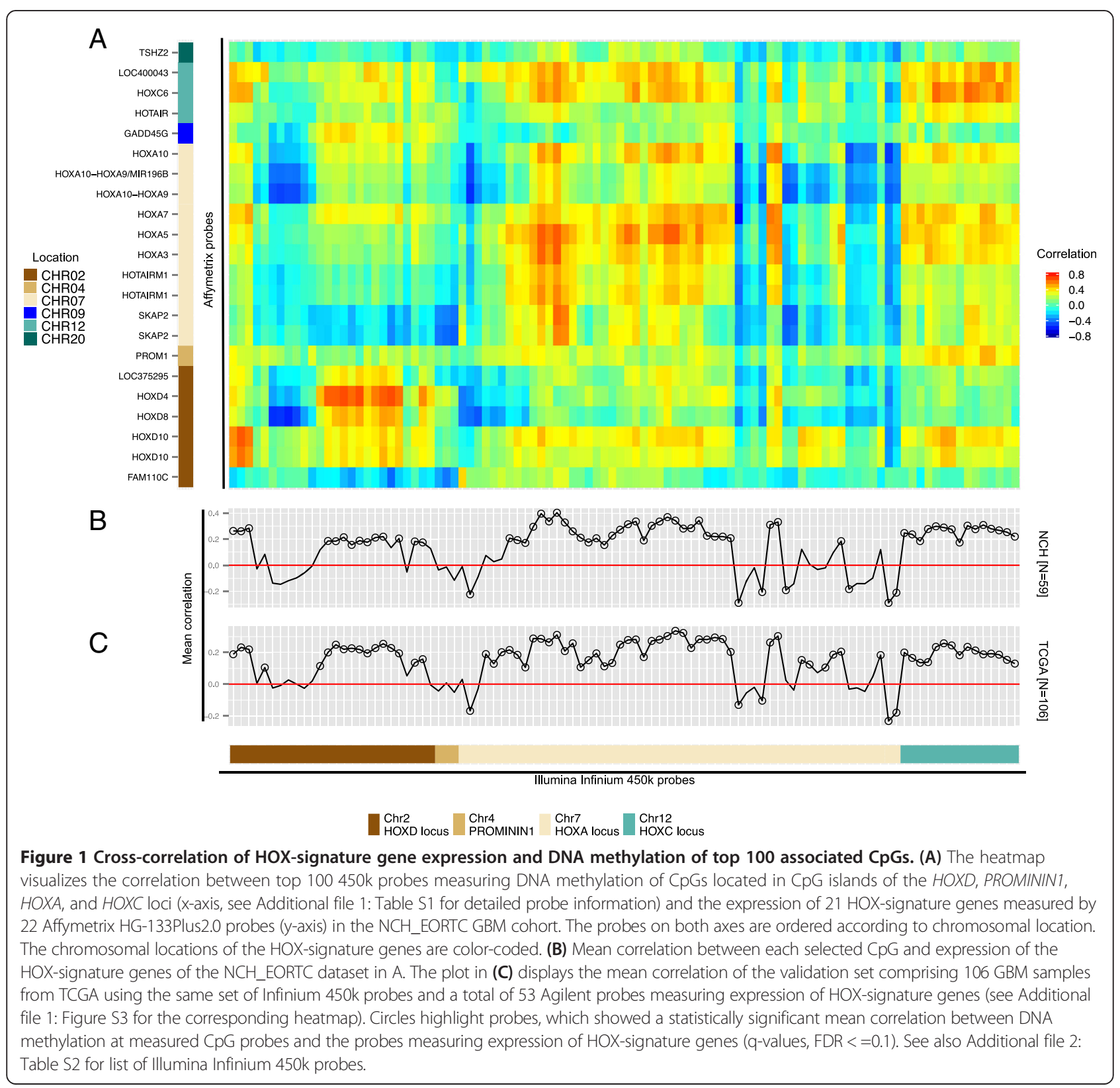


The heatmap of the correlation matrix (Figure 1A) visualizes the pattern of negative and positive correlation between DNA methylation and gene expression in the $\mathrm{NCH}$ EOORTC dataset. The strongest mean negative correlation $(<=-0.28,(-0.28 ; 0.4))$ between expression of HOX-signature genes and methylation was observed for a CpG probe located in the CGI associated with the alternative transcription start site of HOXA10 (probe ID cg05092861 in UCSC CpG Island CHR7: 2721930927219750, FDR <0.10) (Figure 1B, Additional file 2: Table S2). An adjacent CpG showed a similar correlation (cg01078824; -0.21 , FDR $<0.10$; Additional file 2: Table S2).

\section{Determination of the correlation signature in an independent GBM dataset}

Likewise we calculated the correlation matrix between DNA methylation and gene expression in an independent dataset of 106 GBM from The Cancer Genome Atlas (TCGA; Additional file 1: Table S4; mean correlation Figure 1C) visualized in a heatmap in Additional file 1: Figure S3. The similarity of the structure of the correlation matrices was remarkable for these two independent GBM datasets, with an RV-coefficient of 0.84 (simulated $P$ value $<0.001$ (9,999 permutations), Additional file 1: Figures S3 and S4). Of note, while DNA methylation data was also generated on the $450 \mathrm{k}$ platform (used as the common dimension), the expression data originated from different platforms. In the GBM TCGA Agilent data the HOX-signature genes are covered with 53 probes (Additional file 1: Table S5), with probes missing for the ncRNA genes MIR1OB and HOTAIRM1.

In order to evaluate the relevance of our findings in the context of the whole CHR7, we tested whether the apparent local enrichment of negative correlation between HOXA gene expression and DNA methylation at the HOXA locus was statistically significant. We determined the negative correlations between 711 RefSeq annotated CHR7 genes and their respective Illumina Infinium $450 \mathrm{k}$ probes and plotted the values according to genomic location of the genes (Additional file 1: Figure S5A). Gene set enrichment analysis (GSEA) revealed that the 10 HOXA genes were significantly enriched when testing their positions in the ranked list of the observed correlation coefficients of the 711 CHR7 genes as visualized in Additional file 1: Figure S5B ( $P$ value $<0.001$, Additional file 1: Table S3). A similar result was obtained for the TCGA dataset $(P$ value $<0.001$; Additional file 1 : Figure S5C; Table S3).

\section{The relationship DNA methylation/gene expression depends on CpG location}

Next we were interested to evaluate the relationship of the mean correlation methylation/HOX-signature expression
(Figure $1 \mathrm{~B}$ and $\mathrm{C}$ ) and the structural location of the respective CpGs using the Illumina annotation of the 450k probes (1st Exon, 3'UTR, 5'UTR, gene body, transcription start site (TSS) 1,500, TSS200). We observed that negative correlations between DNA methylation and the HOX-signature expression are primarily found for probes which are located either in the 1st exon of a gene, or within $200 \mathrm{bp}$ of TSS, in line with canonical effects of promoter-CGI methylation on gene expression, while positive mean DNA methylation/gene expression correlations were found for probes located in the 3'UTR, 5'UTR, gene body and within 1,500 bp of TSS. This observation was consistent between the NCH_EORTC and the TCGA dataset (Additional file 1: Figure S6A and B, $P$ value $<0.01$ and $P$ value $<0.05$, one-way ANOVA).

\section{DNA methylation at HOXA10 promoter CGI is lower in HOX-high than HOX-low GBM}

The 59 GBM (NCH_EORTC) were classified into HOXhigh $(\mathrm{n}=25)$ or low $(\mathrm{n}=34)$ based on iterative $k$-means clustering of the 22 Affymetrix probesets (Additional file 1: Figures S7 and S8). The average expression of the HOX-signature in the HOX-low group is not significantly different from respective measures in nontumoral brain samples ( $P$ value $=0.9$, all Welch's Twosample t-test) (Additional file 1: Figure S9A). In contrast, the higher mean expression levels of the HOX-signature in HOX-high samples are significantly different to both HOX-low $(P$ value $<0.01)$ and non-tumoral brain samples $(P$ value $<0.01)$. We observed significant differences in the degree of DNA methylation measured for probe cg05092861 between HOX-low and HOX-high samples, with a higher level of DNA methylation in HOX-low samples $(P$ value $<0.001)$. Both were different from nontumoral brain $(\mathrm{n}=4)$, which showed the lowest methylation levels $(P$ value $<0.001)$, although no expression is detected, while highest levels of DNA methylation were measured in HOX-low samples (non-tumoral brain < HOX-high $<$ HOX-low, Additional file 1: Figure S9B). Similar differences were observed for the adjacent Infinium 450k probe cg01078824.

\section{Correlation between expression and gene copy number}

In order to investigate our hypothesis that gene dosage may contribute to the aberrant expression of the HOXsignature, we first tested the overall relationship between gene dosage and gene expression in our GBM dataset (NCH_EORTC). At the resolution of chromosomal arms that averages out regulatory factors affecting individual transcript levels, expression of the genes located on that arm and corresponding DNA copy number are strongly correlated (median Pearson correlation coefficient 0.73, standard deviation 0.18). The gene dosage effect on expression is particularly striking for gain of CHR7 and loss 
of CHR10, both hallmarks of GBM, as visualized in Figure 2 for the whole NCH-EORTC cohort (Figure 2A and $\mathrm{B}$ ), or an individual GBM samples (Figure $2 \mathrm{C}$ and $\mathrm{D}$ ).

\section{Gene dosage and DNA methylation impact HOX-signature expression}

Next we tested the association between copy number alterations (CNA) at cytoband 7p15.2 that harbors the HOXA locus, methylation at the selected CpG (cg05098261) in the alternative promoter of HOXA10, and mean HOXsignature expression levels using an explanatory linear model. The M-values of the probe cg05092861 and the respective CNA values for cytoband 7p15.2 were introduced as independent variables, while the mean HOX-signature expression (based on scaled and centered gene expression) was the dependent variable. We observed a significant additive effect between CNA 7p15.2 and methylation at the selected probe in the alternative promoter of HOXA10 as illustrated in Figure 3A. This model explains 32\% of the variance of the HOX-signature expression levels (Table 1 'Model 1'). The result supports the hypothesis that DNA methylation - at least in part - acts as an attenuating factor in samples with increased CNA at 7p15.2 in a manner that the effect of increased gene dosage on expression may be countered by the inhibitory effect of DNA methylation of HOXA10 in GBM. Similar results were also observed in the TCGA subset of 103 GBM (for 3 of the 106 TCGA GBM the SNP6 CNA data were not available) as visualized in Figure 3B and summarized in Table 1. The model for the TCGA data explains $29 \%$ of the observed variance in HOX-signature expression (Table 1 - 'Model 1'). Similar additive effects were observed for the adjacent CpG (cg01078824, Table 1 - 'Model 2').

Given the apparent central role of HOXA10 in the HOX-signature, X/Y plots for both series of GBM, stratified by CHR7 status, illustrate the correlation between HOXA10 expression alone (Affymetrix probe 214651_s_at) and methylation at the above identified 2 top CpGs (cg05092861, cg01078824) in the promoter of the putative non-coding alternative HOXA10 transcript (NR_037939.1) and in addition at two CpGs (cg18243072, TSS200; cg14625175, exon1) located in the CGI of the

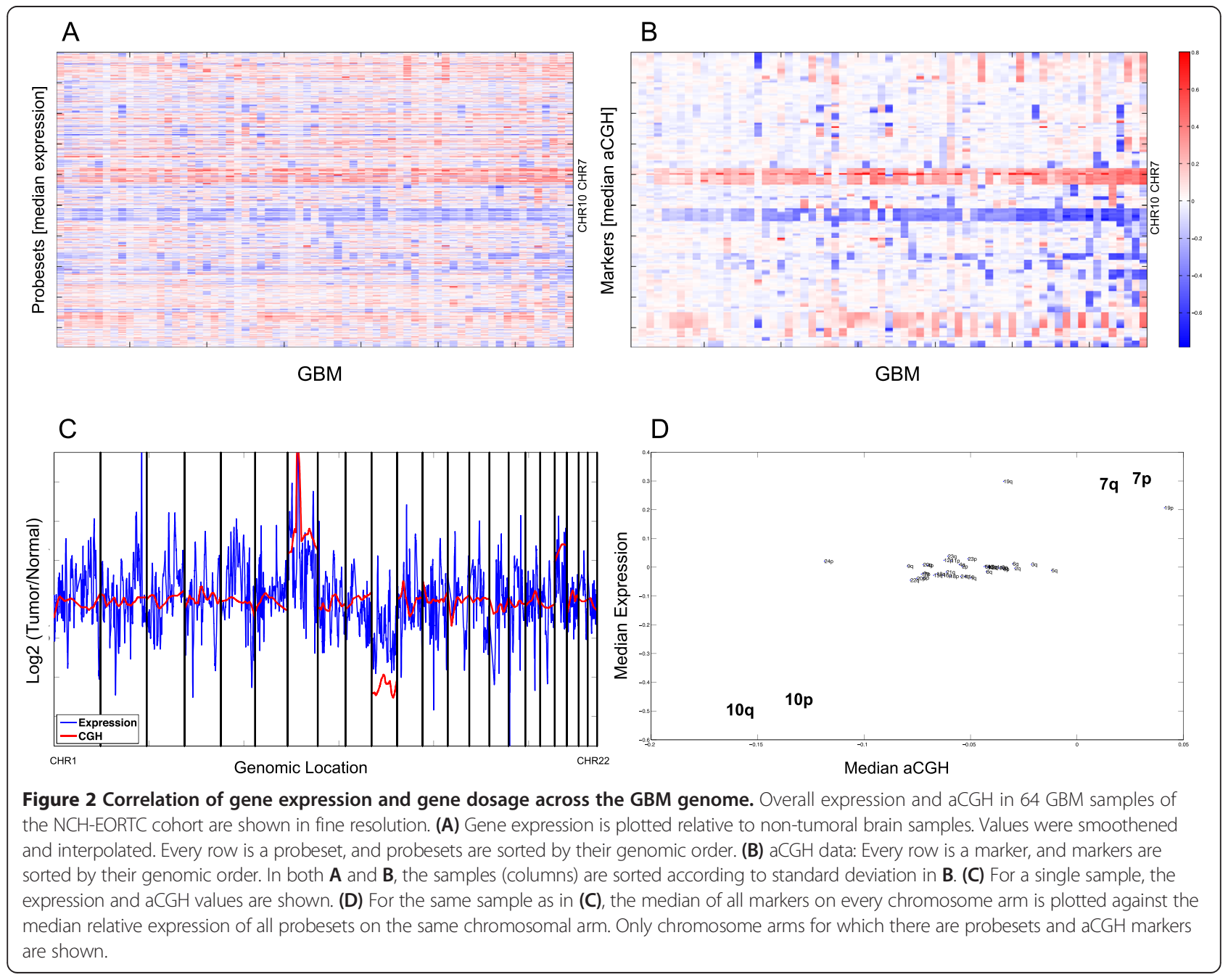




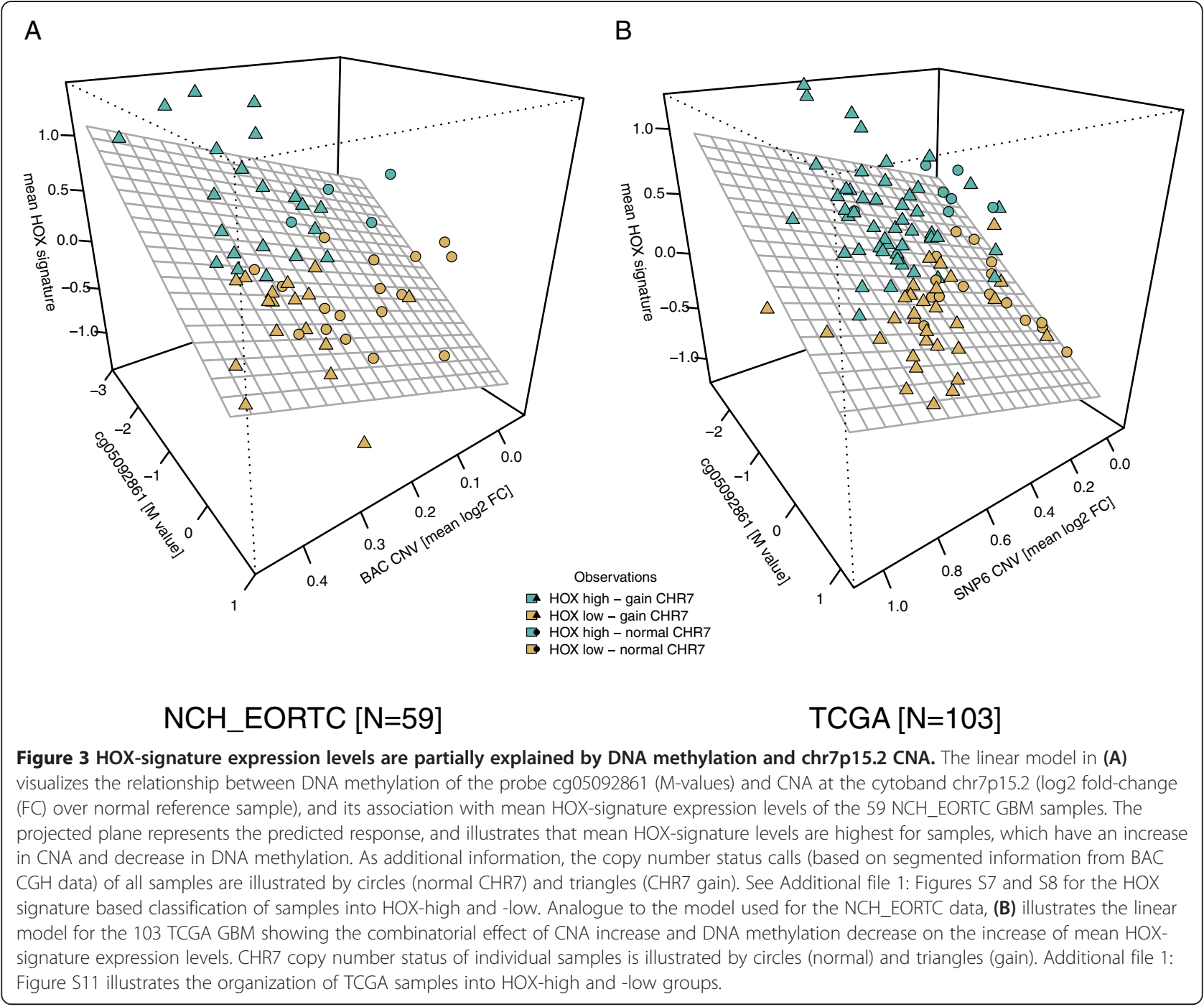

promoter of the canonical HOXA10 protein-coding transcript (NM_018951.3) (Figure 4B and C). In our dataset (NCH_EORTC) a significant negative correlation between expression and methylation was associated with CpG methylation in both regions for HOXA10 expression in GBM with CHR7 gain (Spearman's correlation $P<0.05$, Figure $4 \mathrm{~B}$ and $\mathrm{C})$. In contrast, no significant correlation was observed in GBM with normal CHR7 status (Figure 4B and $\mathrm{C}$ ). Taken together, the findings for the HOX-signature gene $H O X A 10$ are in accordance with the model presented in Figure 3. Similar results were obtained for the TCGA dataset (Additional file 1: Figure S10B and C). The Spearman's correlation coefficients for CpGs located in the two HOXA10 promoters and HOXA10 expression are available in Additional file 1: Table S6 for both datasets, stratified by CHR7 status. Additional file 1: Table S7 comprises the correlation coefficients for the top $100 \mathrm{CpGs}$ and mean expression of the whole HOX-signature, stratified by $\mathrm{CHR7}$ status for both NCH_EORTC and TCGA GBM.
Transcriptome at the HOXA locus in GBM derived sphere (GS) lines

We sought to extend our findings to four GBM-derived sphere lines (GS lines) that retain GBM-relevant stemcell properties and provide a better model of GBM than regular cell lines. Aligned read pile-ups derived from directed RNA-Seq data shown in Figure 5 illustrate the presence of both sense and anti-sense transcripts in the HOXA locus in three GS lines (LN-2207GS, LN-2669GS, LN-2683GS, thus designated as HOX-high models). In contrast, RNA-Seq reads generated from the HOX-low GS line LN-2540GS and human brain tissue (Ensembl Illumina Human BodyMap 2.0) show only very few and no aligned reads across the HOXA locus, respectively. These data confirm that the expression of transcripts from the HOXA locus in HOX-high GS lines is abnormal when compared to adult 'normal' brain. In the three HOX-high GS lines, the visualization of read alignments show that the majority of fragments originated from 
Table 1 Coefficients of linear models for HOX-signature expression in GBM datasets

\begin{tabular}{|c|c|c|c|c|c|c|}
\hline Coefficients & Estimate & Std. Error ${ }^{a}$ & t value $^{b}$ & $\operatorname{Pr}(>|t|)^{c}$ & $R^{2 d}$ & VIF $^{\mathrm{e}}$ \\
\hline NCH_EORTC (Model 1) & & & & & 0.318 & \\
\hline (Intercept) & -0.793 & 0.166 & -4.791 & $1.26 \mathrm{e}-05$ & & \\
\hline $\operatorname{cg} 05092861$ & -0.453 & 0.110 & -4.137 & 0.0001 & & 1.001 \\
\hline CNA chr7p15.2 (BAC) & 1.804 & 0.554 & 3.257 & 0.0019 & & 1.001 \\
\hline NCH_EORTC (Model 2) & & & & & 0.215 & \\
\hline (Intercept) & -0.204 & 0.168 & -1.212 & 0.231 & & \\
\hline cg01078824 & -0.311 & 0.113 & -2.754 & 0.008 & & 1.002 \\
\hline CNA chr7p15.2 (BAC) & 1.822 & 0.594 & 3.066 & 0.003 & & 1.002 \\
\hline TCGA (Model 1) & & & & & 0.289 & \\
\hline (Intercept) & -0.612 & 0.105 & -5.822 & 7.07e-08 & & \\
\hline cg05092861 & -0.372 & 0.067 & -5.542 & $2.44 \mathrm{e}-07$ & & 1.000 \\
\hline CNA chr7p15.2 (SNP6) & 0.664 & 0.184 & 3.608 & 0.000 & & 1.000 \\
\hline TCGA (Model 2) & & & & & 0.254 & \\
\hline (Intercept) & -0.158 & 0.088 & -1.785 & 0.077 & & \\
\hline cg01078824 & -0.356 & 0.071 & -4.962 & $2.87 e-06$ & & 1.023 \\
\hline CNA chr7p15.2 (SNP6) & 0.799 & 0.191 & 4.186 & $6.13 e-05$ & & 1.023 \\
\hline
\end{tabular}

${ }^{\mathrm{a}}$ Standard error.

bt-statistics.

${ }^{\mathrm{C}}$ Two-sided $P$ values of t-statistic.

${ }^{\mathrm{d}} \mathrm{R}$-squared provides information about the variance explained by the model.

e Variance inflated factor (VIF), provides information about multi-collinearity of the model variables.

exons of HOXA10, with presence of some transcripts for HOXA9 exons. We then used the RNA-Seq data to perform reference annotation based transcript assembly using cufflinks. This mapped transcript fragments of the three HOX-high GS lines to 12 of the 15 canonical HOXA sense and anti-sense transcripts. Additionally, using transcriptome re-assembly we found that the mapping of some of the transcript fragments suggests that a read-through transcript may be present, possibly consisting of exons of HOXA10, miR-196b, HOXA9, and the putative protein-coding RNA RP1-170019.20. The presence of the AFFY-probes 214651_s_at and 209905_at in the HOX-signature that have been annotated as long non-coding RNA gene HOXA10-HOXA9 lend some support to these findings. A model of this putative readthrough transcript, based on the cufflinks reconstruction of the transcriptome of the GS lines, is visualized in the HOXA locus displayed in Figure 5. A Sashimi plot of the HOXA10-9 locus (Additional file 1: Figure S12) illustrates the presence of transcript fragments, which span the exon/exon junctions of HOXA9, HOXA10, miR-196b, and RP1-170O19.20. However, further studies are warranted.

Histone marks and promoter methylation at the HOXA10/ 9 promoters

One important regulatory mechanism of transcription is the conformational state of chromatin, as mediated through various post-translational histone modifications.
The observation that HOXA genes are actively transcribed in a subset of GBM and in three of our four GS lines indicates that the chromatin is in a permissive state. We tested this assumption by performing chromatin-immunoprecipitation (ChIP) in the four GS lines for three different histone marks: H3K4me3 - mark for active or poised promoters; H3K36me3 - indicative of transcriptional elongation; and H3K27me3 - a repressive mark, followed by $\mathrm{qPCR}$ at the promoters of HOXA10 and HOXA9 (Figure 6A and B). ChIP-qPCR revealed a predominant signal for $\mathrm{H} 3 \mathrm{~K} 4 \mathrm{me} 3$ and presence of H3K36me3 in the HOX-high GS lines (LN-2207GS, LN-2669GS, LN-2683GS) in the HOXA10 promoter, while they were absent in the HOX-low GS line LN2540GS. In contrast, the predominant mark detected in the HOXA9 promoter was H3K36me3, while the other marks were absent. In addition, we determined the methylation status using methylation-specific (MS) clone sequencing of the $\mathrm{CpG}$ island of the HOXA10 promoter (CHR7:27212417-27214396) in three GS lines (2 HOXhigh and 1 HOX-low) and their respective original GBM. The methylation pattern in the GS lines was highly similar to the original primary tumors from which they were isolated, excluding an in vitro artifact (Figure $6 \mathrm{C}$ and $\mathrm{D}$ ). The HOXA10 promoter was unmethylated in the two HOX-high GS lines LN2207GS and LN-2669GS and their respective original GBM, and fully methylated in the HOX-low GS line 


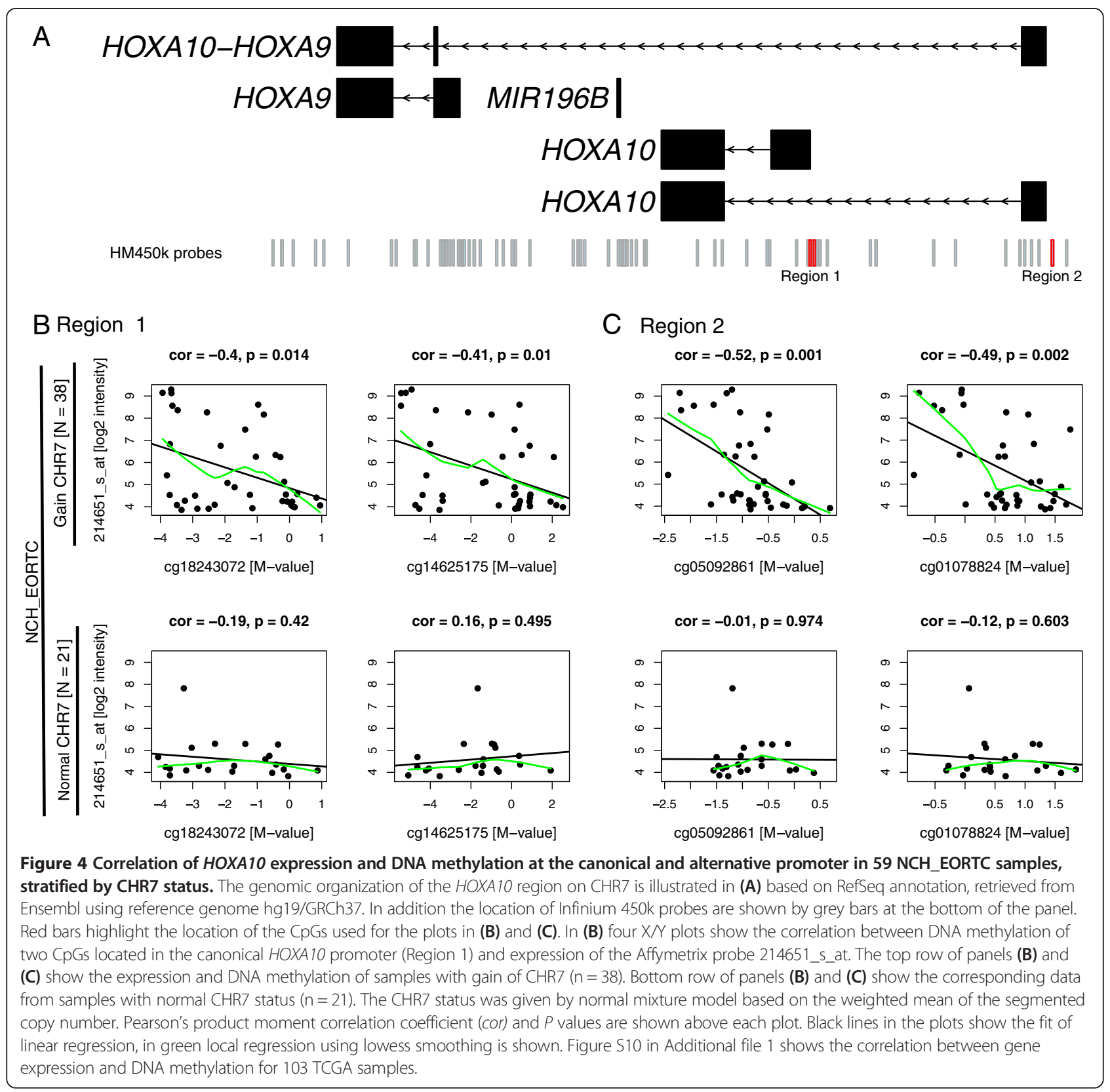

LN-2540GS and the respective GBM-2540. This corroborates our finding of strong negative correlations between expression/methylation for 2 CpGs (cg18243072, cg14625175) interrogated in this region on the $450 \mathrm{k}$ BeadChip, as illustrated and annotated in X-Y-plots for both NCH_EORTC (Figure 4) and TCGA samples (Additional file 1: Figure S10). MS clone sequencing of the HOXA9 promoter (CHR7:27205048-27205315) revealed full methylation also in the HOX-high GS lines (Figure 6E). Taken together, the marks for actively transcribed chromatin are in accordance with HOXA10 expression. HOXA10 is also translated into protein as we have shown previously for GS lines, including LN-
2207GS and a series of GBM on a tissue micro array [7]. The observed enrichment for the elongation mark H3K36me3 at the fully methylated HOXA9 promoter would be compatible with the presence of a putative read-through transcript of HOXA1O and HOXA9, as suggested by our analyses of the RNA-Seq data (Figure 5 and Additional file 1: Figure S12) and the probe annotation from the HG-133Plus2.0 GeneChip.

\section{HOX-signature associated microRNAs}

To test for the potential impact of regulatory microRNAs on the HOX-signature we analyzed the correlation between the mean expression of the HOX-signature in the 


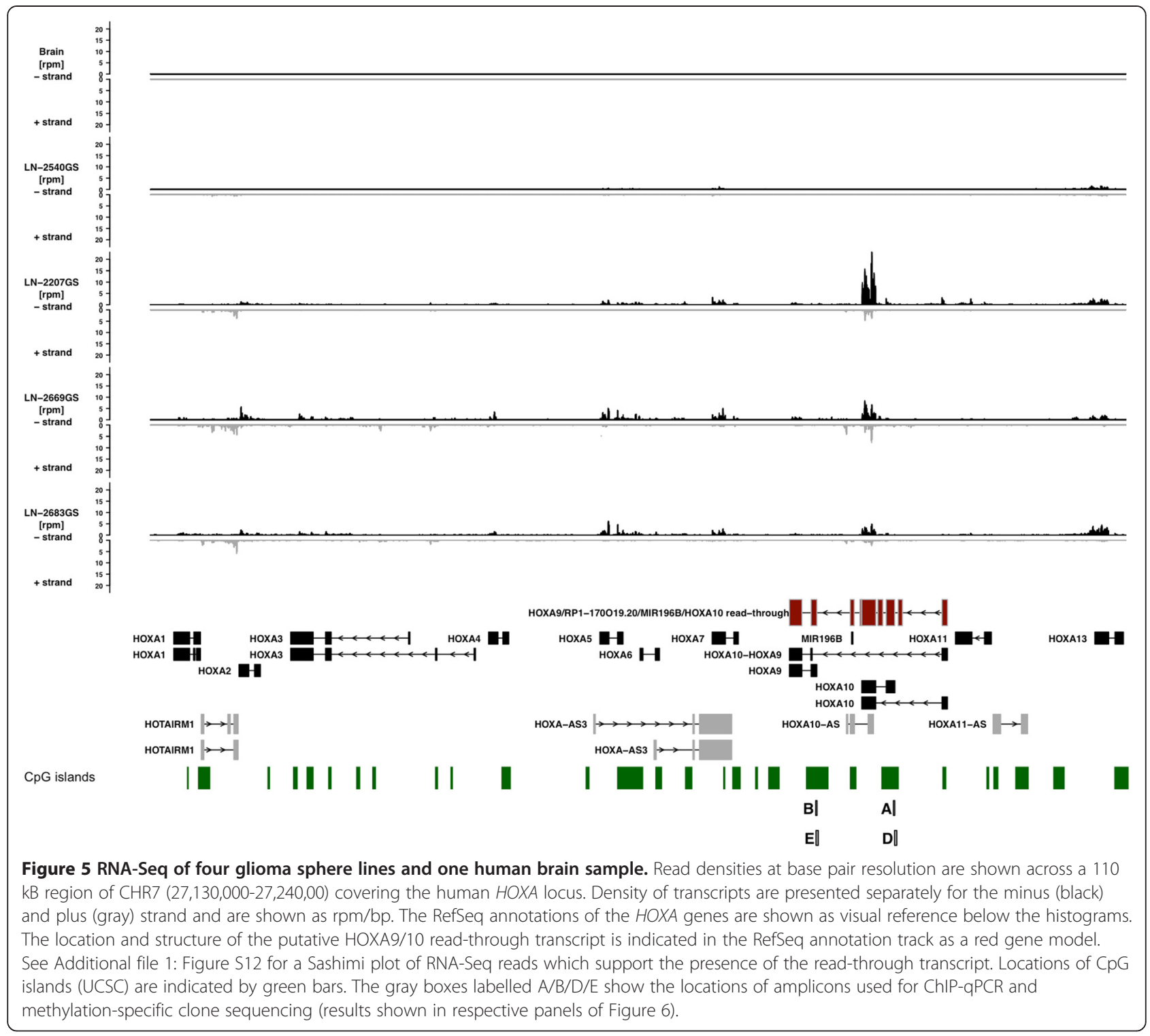

TCGA Agilent data subset and the expression levels of individual microRNAs (Table 2). We found that the top correlated microRNAs (Spearman's rho $>=0.5, P$ value $<0.0001)$ were in fact transcribed from $H O X$ loci (miR-196b - HOXA, miR-10a - HOXB, miR-10b $H O X D)$, known to have regulatory functions in cis [20]. However, the expression of these microRNAs was positively correlated, suggesting that the de-repression of $H O X$ genes in GBM may lead to an overall aberrant co-expression of genes from the affected loci including the enclosed microRNAs. This is further supported by the observation that several long non-coding RNAs transcribed from the HOXA (HOTAIRM1) and HOXC loci (LOC400043, HOTAIR) and adjacent to the HOXD locus (LOC375295), were detected as part of the HOXsignature (Additional file 1: Table S1).

\section{Relationship of the HOX-signature and molecular GBM} subtypes

Next we sought to address how the HOX-signature was related to three established molecular GBM classification schemes: (1) the four GBM expression subtypes neural, proneural, mesenchymal, and classical as proposed by Verhaak and colleagues [21]; (2) the glioma CpG island methylator phenotype (G-CIMP) present in a subgroup of proneural GBM [22]; or (3) distinction of MGMT promoter methylated vs. unmethylated that has been shown to be highly predictive for benefit from alkylating agent chemotherapy [23]. Expression data from 473 GBM (TCGA, level 2 Agilent) were used to classify samples into either HOX-high (259) or low (214), based on k-means clustering (Additional file 1: Figure S13), and were annotated with the expression subtype 


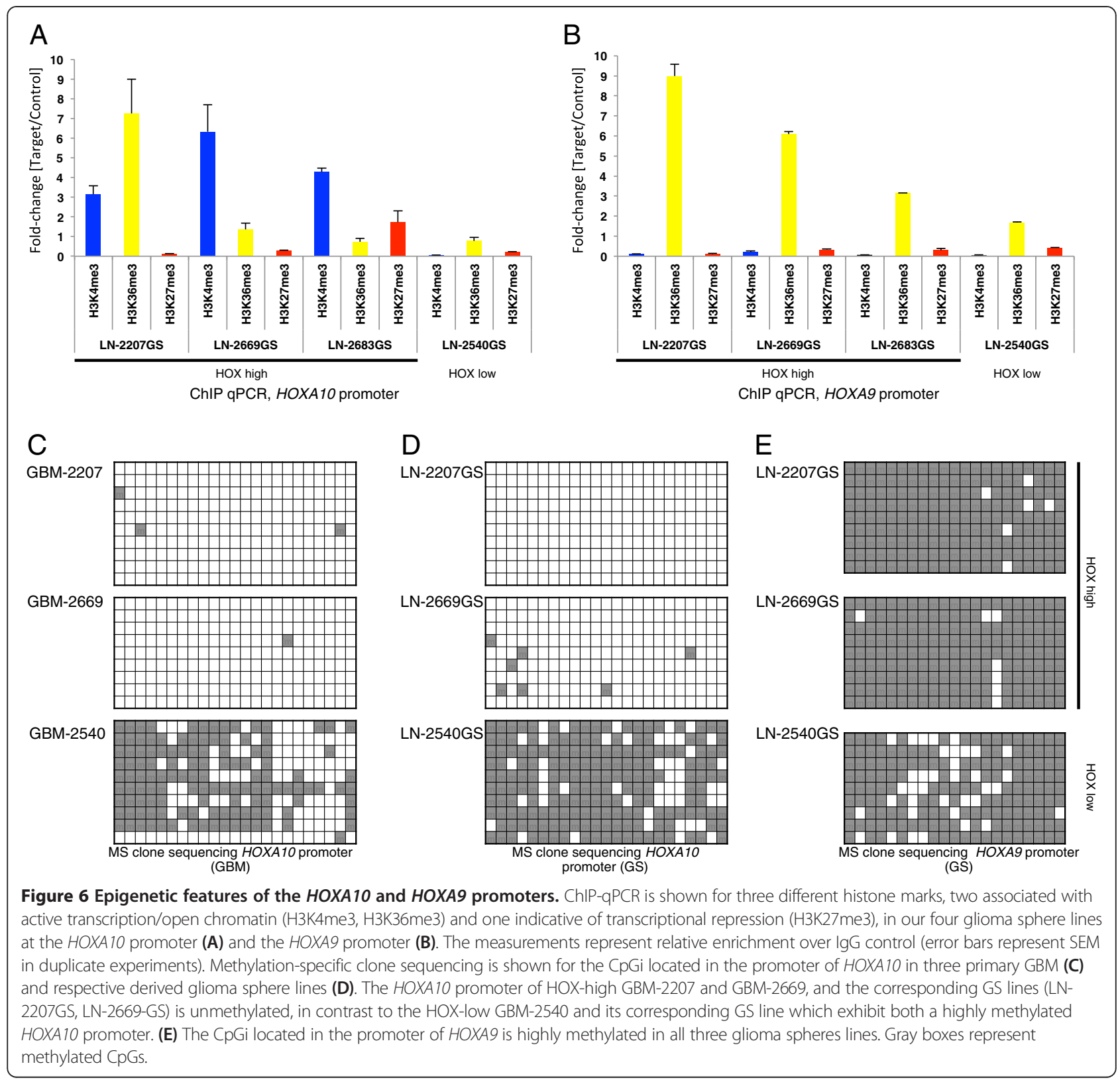

classification, including G-CIMP, and MGMT promoter methylation status [2,24] (Additional file 1: Figure S14). We observed an enrichment of proneural GBM in the HOX-high group, while the proneural G-CIMP-positive GBM were under-represented (Additional file 1: Table S8, $P$ value $<0.001$, Pearson's Chi-squared test). No significant associations were found in the other three expression subtypes. No correlation was found with expression of PDGF that has been proposed as a gain of CHR7-associated driver gene for G-CIMP negative GBM [19]. Finally, we confirmed our previous finding from the NCH_EORTC dataset [7] that the MGMTpromoter methylation frequency was not different between the HOX-high and low groups (Additional file 1: Table S9, $P$ value $>0.35$, Pearson's Chi-squared test).

\section{Discussion}

In the present study we sought to elucidate underlying molecular mechanisms triggering the inappropriate expression of a HOX-signature. Such a HOX gene dominated expression signature has been associated by others, and us with resistance to temozolomide and conferring of glioma-initiating properties $[7,8,11,12]$.

The hypermethylation of the HOXA locus was associated with gain of CHR7, which is reminiscent of compensation for increased gene dosage, known from 
Table 2 Correlation between microRNAs and mean HOXsignature expression in 106 GBM samples from TCGA, top 2 percentile positively and negatively correlated microRNAs

\begin{tabular}{lll}
\hline & Spearman's rho & $\begin{array}{l}\boldsymbol{P} \text { value } \\
\text { (two-sided) }\end{array}$ \\
\hline hsa-miR-196b (HOXA locus) & 0.69 & $<0.01$ \\
hsa-miR-10b (HOXD locus) & 0.50 & $<0.01$ \\
hsa-miR-196a (HOXB/C locus) & 0.5 & $<0.01$ \\
hsa-miR-148a & 0.42 & $<0.01$ \\
hsa-miR-106b & 0.35 & $<0.01$ \\
hsa-miR-25 & 0.32 & $<0.01$ \\
hsa-miR-496 & 0.31 & $<0.01$ \\
hcmv-miR-UL148D & 0.3 & $<0.01$ \\
hsa-miR-337 & 0.29 & $<0.01$ \\
hsa-let-7c & 0.29 & $<0.01$ \\
hsa-miR-130b & 0.29 & $<0.01$ \\
hsa-miR-199a* & -0.27 & $<0.01$ \\
hsa-miR-223 & -0.27 & $<0.01$ \\
hsa-miR-148b & -0.28 & $<0.01$ \\
hsa-let-7f & -0.29 & $<0.01$ \\
hsa-miR-125a & -0.29 & $<0.01$ \\
hsa-let-7d & -0.3 & $<0.01$ \\
hsa-miR-98 & -0.3 & $<0.01$ \\
hsa-let-7 g & -0.33 & $<0.01$ \\
hsa-miR-143 & -0.36 & $<0.01$ \\
hsa-miR-107 & -0.37 & $<0.01$ \\
hsa-miR-145 & -0.42 & \\
\hline L & & $<.01$ \\
\hline
\end{tabular}

Lines in bold highlight microRNAs transcribed from HOX loci.

$\mathrm{X}$-chromosome inactivation [25-28]. This is further supported by the observation that a significant correlation between DNA methylation and expression was only observed for samples with gain of $\mathrm{CHR} 7$ as visualized for HOXA10. The involvement of gene dosage mediated induction of $\mathrm{HOX}$-signature expression is compatible with the observation that HOX-high GBM are under-represented in G-CIMP positive GBM, which reportedly have a much lower frequency of CHR7 gain [2]. The observed correlation between expression of the whole HOX-signature and DNA methylation suggested DNA methylation patterns permissive for expression. However, the regulatory effects leading to coordinated expression of $H O X A, C$, and $D$ genes, and the other members of the signature, including the stem cell marker PROM1 that are located on other chromosomes, are not yet explained. With the exception of the developing forebrain where $H O X$ genes are repressed [29], the coordinated expression and silencing of $H O X$ genes is well known from embryonic development. It involves changes of higher chromatin organization and complex regulation implicating longrange control mechanisms which are only partly understood [14].

HOX genes are tightly regulated through polycombrepressor complex 2 (PRC2)-mediated tri-methylation of H3K27 [30]. Investigation of GS lines suggested loss of the repressive mark H3K27me3 and gain of the active mark H3K4me3 in the promoter of HOXA10 proteincoding transcript variant 1 in HOX-high GS lines, and was associated with an unmethylated CGI. This observation is in accordance with the detection of HOXA10 protein in HOX-high GS lines, as well as in a subset of GBM [7]. In contrast, the HOX-low GS line lacked the active mark and displayed a methylated HOXA10 promoter. Interestingly, the histone marks in the HOXA9 promoter displayed enrichment of H3K36me3 in the HOX-high GS lines in conjunction with the fully methylated CGI suggested transcriptional elongation. This pattern of histone marks would also be compatible with presence of a HOXA10/9 read-through transcript, as proposed by the RNA-Seq analysis and the respectively annotated HG-133Plus2.0 probes that are part of the HOX-signature. Little is known about the functional relevance of the putative long non-coding RNA gene HOXA10-HOXA9. It has been proposed as candidate for nonsense-mediated mRNA decay (NMD) [31-33].

Non-coding RNAs, like lincRNAs and microRNAs, can be involved in the regulation of HOX gene expression [34-36]. Our HOX-signature also includes several lincRNAs, which are transcribed from the different $H O X$ loci: LOC400043 and HOTAIR from the HOXC locus, HOTAIRM1 from the HOXA locus, and LOC375295 from the HOXD locus. For the lincRNAs HOTAIR and HOTAIRM1 functions have been investigated. HOTAIRM1 can regulate the expression of HOXA genes through facilitating conformational changes to the chromatin, in proximal distal manner [35-38]. An initial suspicion that HOTAIRM1 and other ncRNAs could be directly involved in the regulation of the HOX-signature genes was tantalizing. However, their expression pattern, and the observation that the top three correlated microRNAs are actually transcribed from the $H O X A / B / C / D$ loci, rather suggested that these small and long ncRNAs are more likely 'caught in the storm' of a coordinated, but inappropriate HOX transcriptional program.

\section{Conclusions}

In conclusion, our results suggest that the aberrant expression of the HOX-signature, which confers stem-cell related properties and resistance to therapy, may be acquired through gene copy gain associated with CHR7 gain. Hypermethylation appears to compensate for gene copy gain at this locus in the HOX-low GBM, preventing CHR7 gain driven increase of expression, while in 
HOX-high GBM key CpGs in the HOXA locus escape hypermethylation. Gene copy gain and methylation at key CpGs in the promoter of HOXA10 putative noncoding transcript variant 2 are strongly associated with the expression of the whole HOX-signature. These findings are remarkably reproducible in an independent GBM dataset from TCGA. The observed mechanism of escape from DNA hypermethylation may explain overexpression of other gliomagenesis relevant protooncogenes located on CHR7 and other loci affected by tumor-related increased gene dosage. Hence, further studies are warranted to investigate the co-evolution of gene copy number changes and epigenetic changes, including tumorigenesis-associated DNA methylation, to identify tumor relevant deregulated genes. Finally, the observation of compensatory DNA methylation at genes with potential proto-oncogenic function should be taken into account when considering epigenetic drugs.

\section{Methods \\ GBM datasets and GBM derived sphere lines}

Our patient cohort of 59 GBM patients (NCH_EORTC), for whom Affymetrix HG-133Plus2.0 gene expression and Illumina Infinium 450k DNA methylation data were available, has been treated within clinical trials $[39,40]$. Patients treated within EORTC 26981 had consented for translational research of their tumor tissues as part of the study protocol. All other patients gave informed consent according to the protocol approved by the local ethics committee (protocol F25/99) and the respective competent Swiss federal authorities (No 1.05.01.10-48). The study protocols conform to the World Medical Association Declaration of Helsinki [41]. Analysis of non-tumoral brain samples and the establishment of the GBM derived sphere lines (GS lines) LN-2207GS, LN-2540GS, LN-2669GS, and LN-2683GS, respective authentication, and the description of the respective original tumors have been published previously $[7,42,43]$. Briefly, GS lines were cultured under stem cell conditions using DMEM-F12 medium (Invitrogen, 10565-018) supplemented with human recombinant EGF and human recombinant basic FGF (Peprotech, AF-100-15 and 100-18B), $20 \mathrm{ng} / \mathrm{mL}$ each, and $2 \%$ B27 (Invitrogen, 17504); 50\% of the medium was substituted twice weekly.

Bisulfite treatment and methylation-specific (MS) clone sequencing, and DNA methylation profiling

DNA isolated from frozen tissues or cells was treated with bi-sulfite, and methylation profiling was performed using Infinium HumanMethylation450 BeadChip (Illumina). MS clone sequencing was performed as previously described [42]. See also Extended Experimental Procedures.

\section{RNA-Seq of glioma sphere transcriptomes and data analysis}

Total RNA isolated from GS cells was depleted from ribosomal RNA and sequencing libraries were prepared using TruSeq Stranded Total RNA with Ribo-Zero Gold (Epicentre, Illumina), followed by paired-end sequencing on Illumina Hiseq (PE 2x50 bp; NXTGNT, University of Gent, Belgium). Details on read-alignment, transcriptome reconstruction and data visualization can be found in the Extended Experimental Procedures.

\section{Chromatin immunoprecipitation followed by quantitative PCR (ChIP-qPCR)}

Chromatin was prepared using the MAGnify Chromatin Immunoprecipitation System (Invitrogen), precipitated with antibodies targeting the interrogated histone marks, and DNA quantified using qPCR as previously described [42]. See Extended Experimental Procedures for details on the procedure and antibodies used for immunoprecipitation.

\section{aCGH dataset}

For the NCH_EORTC samples, the Bacterial Artificial Chromosome (BAC) aCGH data were acquired by UCSF Humarray 2.0 and 3.0 platforms containing 2,428 BACs, each spotted in triplicate, distributed over the human genome with an average resolution of $1.4 \mathrm{Mb}[18,44]$. Details on data processing and analysis are presented in the Extended Experimental Procedures.

\section{Selection of TCGA samples included in the analysis and data processing}

We applied two criteria to select samples from TCGA for our validation dataset: First, the gene expression platform should have sufficient coverage of the HOXsignature in terms of probes measuring expression levels of all 21 genes. Second, DNA methylation should be measured with the Illumina Infinium 450k platform, as this provided us a common dimension necessary to assess the similarity between the two datasets. Details on the sample selection are presented in the Extended Experimental Procedures.

\section{Data analysis}

Unless otherwise stated, all data processing, analysis, and visualization were performed in $\mathrm{R}$ version 3.1.0 [45]. Packages for specific data types and tasks are listed in the relevant sections.

\section{Processing and normalization of Illumina Infinium 450k DNA methylation data}

The methylation array data of samples was loaded into $\mathrm{R}$ and processed using the BioConductor package 'minfi'. The detection $P$-values, probabilities that the target sequence signal was distinguishable from the background, 
were used to exclude probes with poor quality. The probes that are unsuccessfully measured (p-detection $>0.01$ ) in more than $1 \%$ of samples were dropped from the dataset. The DNA methylation data from the $450 \mathrm{k}$ BeadChip were preprocessed as in Genome Studio (software provided by Illumina) and they were summarized by $\mathrm{M}$-values as recommended by Du et al. [46].

\section{Processing and normalization of Affymetrix gene expression data}

The expression intensities for all probe sets from Affymetrix CEL-files were estimated using robust multiarray average (RMA) with probe-level quantile normalization and the Normalized Unscaled Standard Errors values (NUSE) were used to assess the relative quality of arrays. The $\mathrm{R}$ packages affy and affyPLM from BioConductor [47] were used to establish normalization and NUSE values.

\section{Normalization of Agilent gene expression data}

Level 1 Agilent gene expression data were downloaded from the TCGA for 106 samples for which Infinium 450k DNA methylation data were available. The intensities within array were normalized using Loess normalization, followed by quantile normalization between arrays. Missing values were imputed using nearest neighbor averaging method. In a last step, average intensities were calculated for probes, which are present more than once.

\section{HOX classification of samples}

We used the scaled and centered gene expression data for the 22 and 53 probes measuring levels of HOXsignature genes in the NCH_EORTC and TCGA samples, respectively. These data were used as input for an iterative k-means clustering procedures. Parameters were chosen to search for the most stable cluster consisting of two to eight groups of samples, and 10,000 iterations were performed. The number of groups was selected based on which number of clusters had the maximum Calinksi-Harabasz criterion value [48], thus representing the most stable partitioning of samples into groups/clusters. The mean HOX-signature expression levels were then calculated for the different groups and 'high' and 'low' classes were assigned based on the observed mean population-wide expression levels (means of the HOXhigh/-low sample means).

\section{Selection of Illumina Infinium 450k probes}

Probes measuring DNA methylation of the promoters of 21 HOX signature genes were selected based on their annotated location, resulting in a list of 400 probes. To reduce the dimensionality of the DNA methylation data, principle component analysis was performed and only the 100 probes with the highest cumulative contribution retained. Further details on the procedure are presented in the Extended Experimental Procedures.

\section{Correlation between gene copy number and expression}

Expression data and aCGH profiling were available for 64 GBM samples of the NCH_EORTC cohort. For each of those samples, the median aCGH value and the median gene expression value (after each gene was mean centered and divided by the standard deviation of its expression across those samples) were calculated for each of the 39 autosomal chromosome arms. Pearson correlation coefficients between the median aCGH values and median expression values of all chromosomal arms were calculated per sample as described [17].

\section{Correlation between DNA methylation and expression}

Pearson cross-correlation matrices were computed separately to investigate relationship between the filtered methylation data and HOX expression signature datasets for both NCH_EORTC and TCGA samples. A description of the detailed statistical procedure can be found in the Extended Experimental Procedures.

\section{Correlation of TCGA GBM Agilent gene and microRNA expression data}

The correlation between mean HOX-signature gene expression levels and microRNA expression levels were calculated using Spearman's rho statistic to estimate rank-based measure of association, for 96 of the 106 TCGA GBM samples. The top 2 percentile positively and negatively correlated microRNAs were selected for further inspection.

\section{Additive effect of CNA and DNA methylation on mean HOX-signature expression levels}

Mean HOX-signature expression level of each sample was calculated from the scaled and centered expression values of each probe (22 for NCH_EORTC, 53 for TCGA). This value was then designated as the response variable of the linear model. The DNA methylation levels of the probe (M-values) were used as the first explanatory variable, and as a second term the CNA levels as determined by circular binary segmentation at cytoband chr7p15.2 were added (log2 fold-change of tumor over diploid reference):

$$
\begin{aligned}
& \text { Mean }(\text { HOX signature expression }) \sim D N A \text { methylation } \\
& (\text { selected } 450 k \text { probe }[M-\text { value }])+C N A(7 p 15.2[\operatorname{logRR}])
\end{aligned}
$$

\section{Data access}

Gene expression profiles, DNA copy number alteration data (array comparative genomic hybridization (aCGH)) and DNA methylation profiles have in part been 
previously published $[7,24,49]$, and are available in the Gene Expression Omnibus (GEO) database at [50] (accession-number: GSE7696; GSE60507; GSE60274). Due to patient privacy concerns, the RNA-Seq data in the form of raw sequencing data will be made available upon request to the corresponding author, $\mathrm{MEH}$. The molecular profiles of GBM from The Cancer Genome Atlas project (TCGA) were downloaded from $[2,51,52]$.

\section{Additional files}

\section{Additional file 1: Figures S1 to S15, Tables S1 and S3 to S10 and corresponding figure legends, as well as Extended Experimental Procedures in portable document format.}

Additional file 2: Table S2. Mean correlation with HOX signature expression and $P$ values of top 100 selected Infinium 450k probes in $\mathrm{NCH}$ EORTC and TCGA datasets.

\section{Competing interests}

The authors declare that they have no competing interests.

\section{Authors' contributions}

SK participated in the statistical analysis of all datasets and design of the study, performed the bioinformatics analysis of sequencing data, and drafted the manuscript. PB designed and participated in the statistical analysis. DS participated in ChIP-qPCR and MS-Clone-Sequencing experiments of gliomaspheres. IS performed laboratory part of RNA-Seq experiment. TS performed statistical analysis related to correlation of copy number and gene expression in EORTC_NCH dataset. WvC coordinated RNA-Seq experiments and helped to draft the manuscript. IV participated in ChIP-qPCR experiments. RTD, MJB, CM, WPM, and RS participated in collection and assembly of data. ED and MD provided statistical and bioinformatics support. MEH conceived of the study, and participated in its design and coordination, and drafted the manuscript. All authors read and approved the final manuscript.

\section{Acknowledgements}

This work was supported by the Swiss National Science Foundation (3100A-138116), the National Center of Competence in Research Molecular Oncology, and the Swiss Cancer League (KFS-29-02-2012). The work of TS and ED was supported in part by a grant from the Leir Charitable Foundation. The results published here are in part based upon data generated by The Cancer TCGA Genome Atlas pilot project established by the $\mathrm{NCl}$ and NHGRI. Information about TCGA and the investigators and institutions who constitute the TCGA research network can be found at http://cancergenome.nih.gov/ publications/publicationguidelines.

\section{Author details}

${ }^{1}$ Neurosurgery, Lausanne University Hospital, Lausanne 1011, Switzerland. ${ }^{2}$ Neuroscience Research Center, Lausanne University Hospital, Lausanne 1011, Switzerland. ${ }^{3}$ Bioinformatics Core Facility, Swiss Institute for Bioinformatics, Lausanne 1005, Switzerland. ${ }^{4}$ Department of Education and Research, University of Lausanne, Lausanne 1011, Switzerland. ${ }^{5}$ Ben-Gurion University of the Negev, Beersheba, Israel. ${ }^{6}$ Department of Mathematical Modelling, Statistics and Bioinformatics, Ghent University, Ghent, Belgium. ${ }^{7}$ Department of Neurology/Neurooncology, Erasmus MC Cancer Center, Rotterdam, The Netherlands. ${ }^{8}$ Department of Medicine, Medical University Vienna, Vienna, Austria. ${ }^{9}$ Department of Neurology, University of Tübingen, Tübingen, Germany. ${ }^{10}$ Department of Neurology, University Hospital Zurich, Zurich, Switzerland. ${ }^{11}$ Princess Margaret Hospital, University of Toronto, Toronto, Canada. ${ }^{12}$ Department of Physics of Complex Systems, Weizmann Institute of Science, Rehovot, Israel. ${ }^{13}$ Department of Oncology, University Hospital Zurich, Zurich 8091, Switzerland. ${ }^{14}$ Ludwig Center for Cancer Research, University of Lausanne, Lausanne 1011, Switzerland. ${ }^{15}$ Department of Oncology, University of Lausanne, Lausanne 1011, Switzerland. ${ }^{16}$ Present address: The John Curtin School of Medical Research, The Australian National University, Canberra ACT 2601, Australia.
Received: 16 September 2014 Accepted: 8 January 2015

Published online: 27 January 2015

\section{References}

1. Weller M, Pfister SM, Wick W, Hegi ME, Reifenberger G, Stupp R. Molecular neuro-oncology in clinical practice: a new horizon. Lancet Oncol. 2013;14: e370-9.

2. Brennan CW, Verhaak RG, McKenna A, Campos B, Noushmehr H, Salama SR, et al. The somatic genomic landscape of glioblastoma. Cell. 2013;155:462-77.

3. Sottoriva A, Spiteri I, Piccirillo SG, Touloumis A, Collins VP, Marioni JC, et al. Intratumor heterogeneity in human glioblastoma reflects cancer evolutionary dynamics. Proc Natl Acad Sci U S A. 2013;110:4009-14.

4. Singh SK, Hawkins C, Clarke ID, Squire JA, Bayani J, Hide T, et al. Identification of human brain tumour initiating cells. Nature. 2004;432:396-401.

5. Bao S, Wu Q, McLendon RE, Hao Y, Shi Q, Hjelmeland AB, et al. Glioma stem cells promote radioresistance by preferential activation of the DNA damage response. Nature. 2006:444:756-60.

6. Chen J, Li Y, Yu TS, McKay RM, Burns DK, Kernie SG, et al. A restricted cell population propagates glioblastoma growth after chemotherapy. Nature. 2012;488:522-6.

7. Murat A, Migliavacca E, Gorlia T, Lambiv WL, Shay T, Hamou MF, et al. Stem cell-related "self-renewal" signature and high epidermal growth factor receptor expression associated with resistance to concomitant chemoradiotherapy in glioblastoma. J Clin Oncol. 2008;26:3015-24.

8. Gallo M, Ho J, Coutinho F, Vanner R, Lee L, Head R, et al. A tumorigenic MLL-homeobox network in human glioblastoma stem cells. Cancer Res. 2012;73:417-27.

9. Abdel-Fattah R, Xiao A, Bomgardner D, Pease CS, Lopes MB, Hussaini IM Differential expression of HOX genes in neoplastic and non-neoplastic human astrocytes. J Pathol. 2006;209:15-24.

10. Costa BM, Smith JS, Chen Y, Chen J, Phillips HS, Aldape KD, et al. Reversing HOXA9 oncogene activation by PI3K inhibition: epigenetic mechanism and prognostic significance in human glioblastoma. Cancer Res. 2010;70:453-62.

11. Gaspar N, Marshall L, Perryman L, Bax DA, Little SE, Viana-Pereira M, et al. MGMT-independent temozolomide resistance in pediatric glioblastoma cells associated with a PI3-kinase-mediated HOX/stem cell gene signature. Cancer Res. 2010;70:9243-52

12. Kim JW, Kim JY, Kim JE, Kim SK, Chung HT, Park CK. HOXA10 is associated with temozolomide resistance through regulation of the homologous recombinant DNA repair pathway in glioblastoma cell lines. Genes Cancer. 2014:5:165-74.

13. Krivtsov AV, Twomey D, Feng Z, Stubbs MC, Wang Y, Faber J, et al Transformation from committed progenitor to leukaemia stem cell initiated by MLL-AF9. Nature. 2006;442:818-22

14. Montavon T, Duboule D. Chromatin organization and global regulation of Hox gene clusters. Philos Trans R Soc Lond B Biol Sci. 2013;368:20120367.

15. Wigle JT, Eisenstat DD. Homeobox genes in vertebrate forebrain development and disease. Clin Genet. 2008;73:212-26.

16. Alcantara Llaguno S, Chen J, Kwon CH, Jackson EL, Li Y, Burns DK, et al. Malignant astrocytomas originate from neural stem/progenitor cells in a somatic tumor suppressor mouse model. Cancer Cell. 2009;15:45-56.

17. Tsafrir D, Bacolod M, Selvanayagam Z, Tsafrir I, Shia J, Zeng Z, et al. Relationship of gene expression and chromosomal abnormalities in colorectal cancer. Cancer Res. 2006:66:2129-37.

18. Nigro JM, Misra A, Zhang L, Smirnov I, Colman H, Griffin C, et al. Integrated array-comparative genomic hybridization and expression array profiles identify clinically relevant molecular subtypes of glioblastoma. Cancer Res. 2005:65:1678-86

19. Ozawa T, Riester M, Cheng Y-K, Huse JT, Squatrito M, Helmy K, et al. Most humannon-GCIMP glioblastoma subtypes evolve from a common proneural-like precursor glioma. Cancer Cell. 2014;26:288-300.

20. Yekta S, Tabin CJ, Bartel DP. MicroRNAs in the Hox network: an apparent link to posterior prevalence. Nat Rev Genet. 2008:9:789-96.

21. Verhaak RG, Hoadley KA, Purdom E, Wang V, Qi Y, Wilkerson MD, et al. Integrated genomic analysis identifies clinically relevant subtypes of glioblastoma characterized by abnormalities in PDGFRA, IDH1, EGFR, and NF1. Cancer Cell. 2010;17:98-110.

22. Noushmehr H, Weisenberger DJ, Diefes K, Phillips HS, Pujara K, Berman BP, et al. Identification of a CpG island methylator phenotype that defines a distinct subgroup of glioma. Cancer Cell. 2010;17:419-20. 
23. Hegi ME, Diserens AC, Gorlia T, Hamou MF, de Tribolet N, Weller M, et al. MGMT gene silencing and benefit from temozolomide in glioblastoma. $\mathrm{N}$ Engl J Med. 2005;352:997-1003.

24. Bady P, Sciuscio D, Diserens AC, Bloch J, van den Bent MJ, Marosi C, et al. MGMT methylation analysis of glioblastoma on the Infinium methylation BeadChip identifies two distinct $\mathrm{CpG}$ regions associated with gene silencing and outcome, yielding a prediction model for comparisons across datasets, tumor grades, and CIMP-status. Acta Neuropathol. 2012;124:547-60.

25. Bird A. DNA methylation patterns and epigenetic memory. Genes Dev. 2002;16:6-21.

26. Deaton AM, Bird A. CpG islands and the regulation of transcription. Genes Dev. 2011;25:1010-22.

27. Li $E$, Beard $C$, Jaenisch R. Role for DNA methylation in genomic imprinting. Nature. 1993;366:362-5.

28. Suzuki MM, Bird A. DNA methylation landscapes: provocative insights from epigenomics. Nat Rev Genet. 2008;9:465-76.

29. Noordermeer D, Leleu M, Splinter E, Rougemont J, De Laat W, Duboule D. The dynamic architecture of Hox gene clusters. Science. 2011;334:222-5.

30. Margueron R, Reinberg D. The Polycomb complex PRC2 and its mark in life. Nature. 2011:469:343-9.

31. Akiva P, Toporik A, Edelheit S, Peretz Y, Diber A, Shemesh R, et al. Transcription-mediated gene fusion in the human genome. Genome Res. 2006;16:30-6.

32. Mainguy G, Koster J, Woltering J, Jansen H, Durston A. Extensive polycistronism and antisense transcription in the mammalian Hox clusters. PLoS One. 2007;2:e356.

33. Prakash T, Sharma VK, Adati N, Ozawa R, Kumar N, Nishida Y, et al. Expression of conjoined genes: another mechanism for gene regulation in eukaryotes. PLoS One. 2010;5:e13284.

34. Calin GA, Sevignani C, Dumitru CD, Hyslop T, Noch E, Yendamuri S, et al. Human microRNA genes are frequently located at fragile sites and genomic regions involved in cancers. Proc Natl Acad Sci U S A. 2004;101:2999-3004.

35. Rinn JL, Kertesz M, Wang JK, Squazzo SL, Xu X, Brugmann SA, et al. Functional demarcation of active and silent chromatin domains in human HOX loci by noncoding RNAs. Cell. 2007;129:1311-23.

36. Wang KC, Yang YW, Liu B, Sanyal A, Corces-Zimmerman R, Chen Y, et al. A long noncoding RNA maintains active chromatin to coordinate homeotic gene expression. Nature. 2011;472:120-4.

37. Sessa L, Breiling A, Lavorgna G, Silvestri L, Casari G, Orlando V. Noncoding RNA synthesis and loss of Polycomb group repression accompanies the colinear activation of the human HOXA cluster. RNA. 2007;13:223-39.

38. Zhang X, Lian Z, Padden C, Gerstein MB, Rozowsky J, Snyder M, et al. A myelopoiesis-associated regulatory intergenic noncoding RNA transcript within the human HOXA cluster. Blood. 2009;113:2526-34.

39. Stupp R, Dietrich P, Ostermann Kraljevic S, Pica A, Maillard I, Maeder P, et al. Promising survival for patients with newly diagnosed glioblastoma multiforme treated with concomitant radiation plus temozolomide followed by adjuvant temozolomide. J Clin Oncol. 2002;20:1375-82

40. Stupp R, Mason WP, van den Bent MJ, Weller M, Fisher B, Taphoorn MJB, et al. Radiotherapy plus concomitant and adjuvant temozolomide for glioblastoma. N Engl J Med. 2005:352:987-96.

41. World Medical Association. World Medical Association Declaration of Helsinki: Ethical principles for medical research involving human subjects. JAMA. 2013;310:2191-4.

42. Sciuscio D, Diserens AC, van Dommelen K, Martinet D, Jones G, Janzer RC, et al. Extent and patterns of MGMT promoter methylation in glioblastomaand respective glioblastoma-derived spheres. Clin Cancer Res. 2011;17:255-66.

43. Bady P, Diserens AC, Castella V, Kalt S, Heinimann K, Hamou MF, et al. DNA fingerprinting of glioma cell lines and considerations on similarity measurements. Neuro Oncol. 2012;14:701-11.

44. UCSF - array core service - human BAC arrays. [http://cancer.ucsf.edu/research/ cores/array/array-services\#humanBAC]

45. A language and environment for statistical computing. [http://www.R-project.org/]

46. Du P, Zhang X, Huang CC, Jafari N, Kibbe WA, Hou L, et al. Comparison of Beta-value and M-value methods for quantifying methylation levels by microarray analysis. BMC Bioinformatics. 2010;11:587.

47. Bioconductor - open source software for bioinformatics. [http://www.bioconductor.org/]

48. Caliński T, Harabasz J. A dendrite method for cluster analysis. Commun Stat. 1974:3:1-27
49. Lambiv WL, Vassallo I, Delorenzi M, Shay T, Diserens AC, Misra A, et al. The Wht inhibitory factor 1 (WIF1) is targeted in glioblastoma and has a tumor suppressing function potentially by induction of senescence. Neuro Oncol. 2011:13:736-47.

50. Gene Expression Omnibus. [http://www.ncbi.nlm.nih.gov/geo/]

51. The Cancer Genome Atlas Consortium. Comprehensive genomic characterization defines human glioblastoma genes and core pathways. Nature. 2008:455:1061-8.

52. The cancer genome atlas. [http://tcga-data.nci.nih.gov/tcga/tcgaHome2.jsp]

\section{Submit your next manuscript to BioMed Central and take full advantage of:}

- Convenient online submission

- Thorough peer review

- No space constraints or color figure charges

- Immediate publication on acceptance

- Inclusion in PubMed, CAS, Scopus and Google Scholar

- Research which is freely available for redistribution 